\title{
Recurrence Patterns and Timing Courses Following Curative- Intent Resection for Intrahepatic Cholangiocarcinoma
}

\author{
Liang-Shuo Hu, MD, $\mathrm{PhD}^{1}$, Xu-Feng Zhang, $\mathrm{MD}, \mathrm{PhD}^{1,2}$, Matthew Weiss, $\mathrm{MD}^{3}$, Irinel Popescu, $\mathrm{MD}^{4}$, \\ Hugo P. Marques, $\mathrm{MD}^{5}$, Luca Aldrighetti, $\mathrm{MD}^{6}$, Shishir K. Maithel, $\mathrm{MD}^{7}$, Carlo Pulitano, $\mathrm{MD}^{8}$, \\ Todd W. Bauer, MD ${ }^{9}$, Feng Shen, MD ${ }^{10}$, George A. Poultsides, MD ${ }^{11}$, Oliver Soubrane, MD $^{12}$, \\ Guillaume Martel, $\mathrm{MD}^{13}$, B. Groot Koerkamp, MD ${ }^{14}$, Endo Itaru, $\mathrm{MD}^{15}$, and Timothy M. Pawlik, MD, MPH, PhD, \\ FACS, FRACS (Hon) ${ }^{2}$
}

${ }^{1}$ Department of Hepatobiliary Surgery and Institute of Advanced Surgical Technology and Engineering, The First Affiliated Hospital of Xi' an Jiaotong University, Xi' an, China; ${ }^{2}$ Department of Surgery, The Urban Meyer III and Shelley Meyer Chair for Cancer Research, Surgery, Oncology, Health Services Management and Policy, The Ohio State University, Wexner Medical Center, Columbus, OH; ${ }^{3}$ Department of Surgery, Johns Hopkins Hospital, Baltimore, MD; ${ }^{4}$ Department of Surgery, Fundeni Clinical Institute, Bucharest, Romania; ${ }^{5}$ Department of Surgery, Curry Cabral Hospital, Lisbon, Portugal; ${ }^{6}$ Department of Surgery, Ospedale San Raffaele, Milan, Italy; ${ }^{7}$ Department of Surgery, Emory University, Atlanta, GA; ${ }^{8}$ Department of Surgery, Royal Prince Alfred Hospital, University of Sydney, Sydney, NSW, Australia; ${ }^{9}$ Department of Surgery, University of Virginia, Charlottesville, VA; ${ }^{10}$ Department of Surgery, Eastern Hepatobiliary Surgery Hospital, Shanghai, China; ${ }^{11}$ Department of Surgery, Stanford University, Stanford, CA; ${ }^{12}$ Department of Hepatobiliopancreatic Surgery and Liver Transplantation, AP-HP, Beaujon Hospital, Clichy, France;

${ }^{13}$ Division of General Surgery, Department of Surgery, University of Ottawa, Ottawa, ON, Canada; ${ }^{14}$ Department of Surgery, Erasmus University Medical Centre, Rotterdam, The Netherlands; ${ }^{15}$ Gastroenterological Surgery Division, Yokohama City University School of Medicine, Yokohama, Japan

\begin{abstract}
Background. Recurrence of intrahepatic cholangiocarcinoma (ICC) after curative resection is common.

Objective. The aim of this study was to investigate the patterns, timing and risk factors of disease recurrence after curative-intent resection for ICC.

Methods. Patients undergoing curative resection for ICC were identified from a multi-institutional database. Data on clinicopathological and initial operation information,
\end{abstract}

Electronic supplementary material The online version of this article (https://doi.org/10.1245/s10434-019-07353-4) contains supplementary material, which is available to authorized users.

Liang-Shuo $\mathrm{Hu}$ and $\mathrm{Xu}$-Feng Zhang have contributed equally to this study.

(C) Society of Surgical Oncology 2019

First Received: 21 September 2018;

Published Online: 24 April 2019

T. M. Pawlik, MD, MPH, PhD, FACS, FRACS (Hon)

e-mail: tim.pawlik@osumc.edu timing and first sites of recurrence, recurrence management, and long-term outcomes were analyzed.

Results. A total of 920 patients were included. With a median follow-up of 38 months, 607 patients (66.0\%) experienced ICC recurrence. In the cohort, 145 patients $(23.9 \%)$ recurred at the surgical margin, 178 (29.3\%) recurred within the liver away from the surgical margin, 90 (14.8\%) recurred at extraheptatic sites, and 194 (32.0\%) developed both intrahepatic and extrahepatic recurrence. Intrahepatic margin recurrence (median $6.0 \mathrm{~m}$ ) and extrahepatic-only recurrence (median $8.0 \mathrm{~m}$ ) tended to occur early, while intrahepatic recurrence at non-margin sites occurred later (median $14.0 \mathrm{~m} ; p<0.05$ ). On multivariate analysis, surgical margin $<10 \mathrm{~mm}$ was associated with increased margin recurrence (hazard ratio [HR] 1.70, 95\% confidence interval [CI] $1.11-2.60 ; p=0.014)$, whereas female sex (HR 2.12, 95\% CI 1.40-3.22; $p<0.001$ ) and liver cirrhosis (HR 2.36, 95\% CI 1.31-4.25; $p=0.004$ ) were both associated with an increased risk of intrahepatic recurrence at other sites. Median survival after recurrence was better among patients who underwent repeat curative- 
intent surgery (48.7 months) versus other treatments (9.7 months) [ $p<0.001]$.

Conclusions. Different recurrence patterns and timing of recurrence suggest biological heterogeneity of ICC tumor recurrence. Understanding timing and risk factors associated with different types of recurrence can hopefully inform discussions around adjuvant therapy, surveillance, and treatment of recurrent disease.

Surgical resection is the best potentially curative treatment option for patients with intrahepatic cholangiocarcinoma (ICC). ${ }^{1}$ However, long-term prognosis after curative resection remains dismal, with a 5-year survival rate of $20-35 \% .^{2,3}$ The main reason for an unfavorable long-term outcome after resection of ICC is the high incidence of tumor recurrence, which ranges from 50 to $70 \%{ }^{2,4,5}$. While recurrence is common, the biological behavior of ICC tumors can vary. ${ }^{2,4,5}$ Of note, the difference in disease biology and progression, as well as timing and patterns of recurrence, cannot be fully explained or predicted by tumor stage. ${ }^{6,7}$

Management of recurrent ICC can be clinically challenging. While there are scant data in the literature that define treatment of recurrent disease, several investigators have examined the benefits of re-treatment of recurrence. In particular, several groups have reported on varied therapeutic procedures to treat different types of recurrence, including intrahepatic-only, extrahepatic-only and both intrahepatic and extrahepatic recurrent disease. ${ }^{4,8}$ Similar to hepatocellular carcinoma (HCC), ICC most frequently recurs within the liver itself. ${ }^{4,9,10}$ Varying locations of intrahepatic recurrence (e.g. de novo intrahepatic site vs. margin recurrence) might imply different tumor biology and subsequently different disease treatment and postoperative prognosis. Our group and others have previously reported on the time course of any site recurrence after surgery for primary liver cancers, including HCC and ICC. ${ }^{3,9,11}$ Based on data from these previous studies, a 2-year cut-off value has been utilized as the optimal means to differentiate early versus late recurrence. ${ }^{11}$ Interestingly, early recurrence has been associated with tumor characteristics and technical factors, while late recurrence has been associated with underlying liver disease. ${ }^{4,12-16}$ These data serve to emphasize how a more accurate and detailed understanding of the time course associated with different recurrence patterns, as well as the factors predicting specific recurrence patterns, after resection for ICC may be important. Characterizing recurrence following curative intent surgery for ICC can better delineate the risk of recurrence and therefore help tailor postoperative monitoring and perioperative adjuvant treatment strategies. Given the paucity of data available on recurrence following
ICC, and in particular the treatment of different site recurrences, the objective of the current study was to characterize patterns and timing of disease recurrence following resection of ICC using a large, multi-institutional, international database. In addition, we sought to define specific outcomes among patients with different patterns of recurrent ICC.

\section{PATIENTS AND METHODS}

Patients undergoing curative-intent resection for ICC between April 1990 and August 2017 were identified from a multi-institutional database from 15 hepatobiliary centers in North America, Europe, Australia and Asia. Only patients who underwent curative-intent resection for histologically confirmed ICC were included. Resection with curative intent was defined as macroscopic removal of all tumors (R0 or R1 resection). Patients undergoing palliative (R2) resection, ablation only, intra-arterial therapy only or with extrahepatic metastasis were excluded. The Institutional Review Boards of each participating institution approved the study.

\section{Data Collection and Follow-Up}

A standardized datasheet was created for collection of the clinicopathological, and surgical information. Pathologic staging was recoded according to the 8th edition American Joint Committee on Cancer (AJCC) staging guidelines for all study subjects. ${ }^{17}$ Resection margin status was defined as R0 when the tumor margin was microscopically negative, and R1 when the tumor margin was microscopically positive. Satellite lesions were defined as tumors surrounding the main tumor with multiple other satellite nodules or small solitary tumors located near the main tumor that were histologically similar or less differentiated than the main tumor. ${ }^{18}$

After surgery, patients were regularly followed once every 3-4 months within the first 3 years and then once every 6 months until year 5 , after which screening occurred annually. Patients were prospectively monitored for recurrence with serum tumor markers and imaging studies, including ultrasonography, computed tomography (CT) and/or magnetic resonance imaging (MRI). Recurrence was defined as a biopsy-proven recurrent lesion or radiological evidence with cross-sectional imaging plus an elevated cancer antigen (CA) 19-9 level. Disease-free survival (DFS) was defined as the time duration from the date of initial surgery to tumor recurrence, while overall survival (OS) after recurrence was defined as the time duration from the date of recurrence after surgery to patient death or the end of the study, whichever came first. 
Tumor recurrence sites were classified as intrahepatic recurrence at the surgical margin, intrahepatic recurrence other than surgical margin, extrahepatic recurrence only, and intrahepatic plus extrahepatic recurrence based on CT, MRI and/or positron emission tomography (PET)-CT. The initial recurrence site that was identified was utilized for purposes of analysis. Recurrence at the intrahepatic margin was defined as recurrence in the surgical bed or the same segment(s) as the original tumor, whereas intrahepatic recurrence at an 'other' site was defined as recurrent disease in an area other than the operative segment(s). Curative surgical intent therapy for recurrence was defined as macroscopic removal of all recurrent tumors with reresection, ablation, or combined resection plus ablation. Non-curative surgical intent therapy for recurrence was defined as ablation for patients with multiple nodules or resection of liver disease in the setting of extrahepatic disease.

\section{Statistical Analysis}

Continuous variables were expressed as medians with interquartile ranges (IQRs) and were compared using the Student $t$ test or Mann-Whitney $U$ test. Statistical comparisons for categorical variables were made using the Chisquare test or Fisher's exact test. Kaplan-Meier curves were used to estimate median DFS and OS after recurrence, with corresponding 95\% confidence intervals (CIs); the log-rank test was performed for pairwise comparison of recurrence patterns. Factors associated with DFS among patients with different recurrence patterns were identified using univariate and multivariate Cox proportional hazards regression models. Hazard ratios (HRs) and 95\% CIs were estimated. Variables with a $p$ value $<0.05$ on univariate analysis were included in the multivariate models. A twotailed $p$ value $<0.05$ was considered statistically significant. Statistical analyses were performed using SPSS version 21.0 (IBM SPSS Inc., Chicago, IL, USA).

\section{RESULTS}

A total of 920 patients who underwent curative-intent resection for ICC were included in the analytic cohort (Table 1). Median patient age was 59 years (IQR 51-68) and more than half of the patients were male $(n=517$, $56.2 \%)$. Among all patients, $116(12.6 \%)$ patients presented with liver cirrhosis. Median tumor size was $6 \mathrm{~cm}$ (IQR 4.1-8.5) and $162(17.6 \%)$ patients had two or more lesions; 169 (18.4\%) patients underwent an R1 resection. Less than half of patients $(407 / 920,44.2 \%)$ underwent a concomitant lymphadenectomy. Among the 407 patients who had a nodal dissection, $171(42.0 \%)$ patients had at least one lymph node metastasis.

\section{Recurrence Patterns and Disease-Free Survival}

With a median follow-up of 38 months, $607(66.0 \%)$ patients experienced tumor recurrence following resection, while $313(34.0 \%)$ patients had no evidence of recurrence at the time of last follow-up. Among the entire analytic cohort, $380(41.3 \%)$ patients died of tumor recurrence, while $57(6.2 \%)$ patients died of other causes; 227 (24.7\%) patients were alive with recurrent disease, while 256 (27.8\%) were alive free of tumor recurrence. Median, 1-, 3 -, and 5-year DFS for the entire cohort was 17.1 months (95\% CI 14.2-19.7), 57.5, 34.5, and 29.5\%, respectively, while median, 1-, 3-, and 5-year OS was 38.6 months (95\% CI 33.9-44.1), 81.3, 51.5, and 25.5\%, respectively.

Among the 607 patients who recurred, 323 (53.2\%) patients experienced intrahepatic-only recurrence. Among these patients, $145(23.9 \%)$ recurred at the surgical margin, while $178(29.3 \%)$ recurred at a different site within the liver. In contrast, 90 (14.8\%) patients had extrahepatic-only recurrence and $194(32.0 \%)$ patients developed both intrahepatic and extrahepatic recurrence. Extrahepatic recurrent disease was noted most often in the lungs $(n=55)$; other extrahepatic recurrent sites included lymph nodes $(n=46)$, peritoneum $(n=34)$, bone $(n=13)$, and adrenal $(n=5)$. Among the 194 patients who had intrahepatic and extrahepatic recurrence, $134(69.1 \%)$ patients had intrahepatic disease at a location away from the initial surgical margin, while $60(30.9 \%)$ patients had intrahepatic lesion at or near the previous surgical margin.

Among the 607 patients who recurred, $374(61.6 \%)$ had tumor recurrence within 12 months, and 506 (83.3\%) patients had tumor recurrence within 24 months after surgery. Of note, recurrence location differed according to the time of recurrence (Fig. 1a). Within the initial 6 months following surgery, intrahepatic margin recurrence $(n=73$, $37.8 \%)$ and concomitant intrahepatic plus extrahepatic recurrence $(n=63,32.6 \%)$ were the two most common recurrence patterns. While the incidence of intrahepatic margin recurrence decreased over time following the first 6 months after surgery, intrahepatic recurrence at other sites gradually increased in the postoperative period (13.0\% in $1-6$ months vs. $35.4 \%$ in $6-12$ months vs. $41.7 \%$ in 12-24 months; $p<0.001$ for trend). Approximately one-half $(n=73,50.3 \%)$ of patients who recurred at or near the surgical margin recurred within 6 months after surgery (Fig. 1b). In contrast, most patients who developed an intrahepatic recurrence at a non-surgical site, as well as patients who had extrahepatic-only or intrahepatic plus extrahepatic recurrence recurred within 12 months after 
TABLE 1 Clinical and pathological characteristics of patients

\begin{tabular}{|c|c|c|c|c|}
\hline Variable & Whole cohort $(n=920)$ & With recurrence $(n=607)$ & Without recurrence $(n=313)$ & $p$ value \\
\hline Age, years [median (IQR)] & $59(51-68)$ & $59(49-67)$ & $60(52-68)$ & 0.072 \\
\hline Men & $517(56.2)$ & $343(56.5)$ & $174(55.6)$ & 0.770 \\
\hline BMI $\left(\mathrm{kg} / \mathrm{m}^{2}\right)$ & $25.1(22.2-27.8)$ & $25.3(22.3-28.2)$ & $24.2(21.8-26.7)$ & 0.003 \\
\hline Liver cirrhosis & $116(12.6)$ & $75(12.4)$ & $41(13.1)$ & 0.839 \\
\hline CA19-9 (units/ml) & $55.0(17.0-308.1)$ & $77.0(18.8-456.0)$ & $51.5(16.5-243.8)$ & $<0.001$ \\
\hline CEA (ng/ml) & $2.4(1.3-4.2)$ & $2.4(1.3-4.3)$ & $2.4(1.5-4.0)$ & 0.418 \\
\hline Tumor size, $\mathrm{cm}$ [median (IQR)] & $6.0(4.1-8.5)$ & $6.5(4.8-9.0)$ & $5.0(3.5-7.4)$ & $<0.001$ \\
\hline Multiple lesions, $\geq 2$ & $162(17.6)$ & $132(21.7)$ & $32(10.2)$ & $<0.001$ \\
\hline Perineural invasion & $122(13.3)$ & $96(15.8)$ & $26(8.3)$ & 0.072 \\
\hline Macrovascular invasion & $99(10.8)$ & $71(11.7)$ & $28(8.9)$ & $<0.001$ \\
\hline Microvascular invasion & $220(23.9)$ & $164(27.0)$ & $56(17.9)$ & 0.172 \\
\hline Direct invasion of adjacent organs & $60(6.5)$ & $54(8.9)$ & $15(3.9)$ & $<0.001$ \\
\hline Biliary invasion & $113(12.3)$ & $86(14.2)$ & $27(8.6)$ & 0.701 \\
\hline Satellite lesions & $191(20.8)$ & $161(26.5)$ & $30(9.6)$ & $<0.001$ \\
\hline AJCC $\mathrm{T}$ category & & & & $<0.001$ \\
\hline $\mathrm{T} 1-2$ & $703(76.7)$ & $464(76.4)$ & $241(77.0)$ & \\
\hline $\mathrm{T} 3-4$ & $141(15.3)$ & $111(18.3)$ & $30(9.6)$ & \\
\hline Missing & $74(8.0)$ & $32(5.3)$ & $42(13.4)$ & \\
\hline Histological grade & & & & $<0.001$ \\
\hline Well to moderately differentiated & $701(76.2)$ & $470(77.4)$ & $231(73.8)$ & \\
\hline Poorly to undifferentiated & $144(15.7)$ & $114(18.8)$ & $30(9.6)$ & \\
\hline Missing & $75(8.2)$ & $23(3.8)$ & $52(16.6)$ & \\
\hline Morphologic type & & & & 0.059 \\
\hline Mass-forming or papillary & $751(71.6)$ & $485(79.9)$ & $266(73.8)$ & \\
\hline Periductal infiltrating \pm mass-forming & $169(18.4)$ & $122(20.1)$ & $45(14.4)$ & \\
\hline Margin (mm) & & & & 0.225 \\
\hline$<1$ & $96(10.4)$ & $73(12.0)$ & $23(7.3)$ & \\
\hline $1-4$ & $325(35.3)$ & $224(36.9)$ & $101(32.3)$ & \\
\hline $5-9$ & $264(28.7)$ & $163(26.9)$ & $101(32.3)$ & \\
\hline$\geq 10$ & $169(18.4)$ & $124(20.4)$ & $45(14.4)$ & \\
\hline Lymphadenectomy & $407(44.2)$ & $303(49.9)$ & $104(33.2)$ & $<0.001$ \\
\hline Lymph node metastasis & $171(42.0)$ & $132(43.7)$ & $39(37.5)$ & 0.009 \\
\hline Major hepatectomy & $495(53.8)$ & $364(60.0)$ & $131(41.9)$ & 0.063 \\
\hline Major vascular resection & $101(11.0)$ & $70(11.5)$ & $31(9.9)$ & 0.760 \\
\hline Bile duct resection & $186(20.2)$ & $112(18.5)$ & $74(23.6)$ & $<0.001$ \\
\hline Intraoperative blood loss (ml) & $400(200-800)$ & $450(200-800)$ & $300(200-600)$ & 0.291 \\
\hline Duration of surgery (min) & $200(120-315)$ & $200(120-310)$ & $177(96-322)$ & 0.318 \\
\hline Postoperative major complications & $124(13.5)$ & $87(14.4)$ & $37(11.8)$ & 0.106 \\
\hline Adjuvant chemo-/radiotherapy & $286(31.1)$ & $231(38.1)$ & 55 (17.6) & $<0.001$ \\
\hline Adjuvant chemotherapy & $283(30.8)$ & $226(37.2)$ & $57(18.2)$ & $<0.001$ \\
\hline Adjuvant radiotherapy & $46(5.0)$ & $35(5.8)$ & $11(3.5)$ & 0.447 \\
\hline
\end{tabular}

Data are expressed as $n(\%)$ unless otherwise specified

$I Q R$ interquartile range, BMI body mass index, CA19-9 carbohydrate antigen 19-9, CEA carcinoembryonic antigen, AJCC American Joint Committee on Cancer

surgery. Of note, the ratio of extrahepatic-only recurrence (14.8\%) versus intrahepatic plus extrahepatic recurrence $(32.0 \%)$ remained relatively constant following surgery $(p=0.59)$.
Of note, a small subset of patients $(n=22,3.6 \%)$ developed a recurrence more than 5 years after surgery. Among these 22 patients, intrahepatic recurrence at the resection bed was noted in five patients, while intrahepatic 


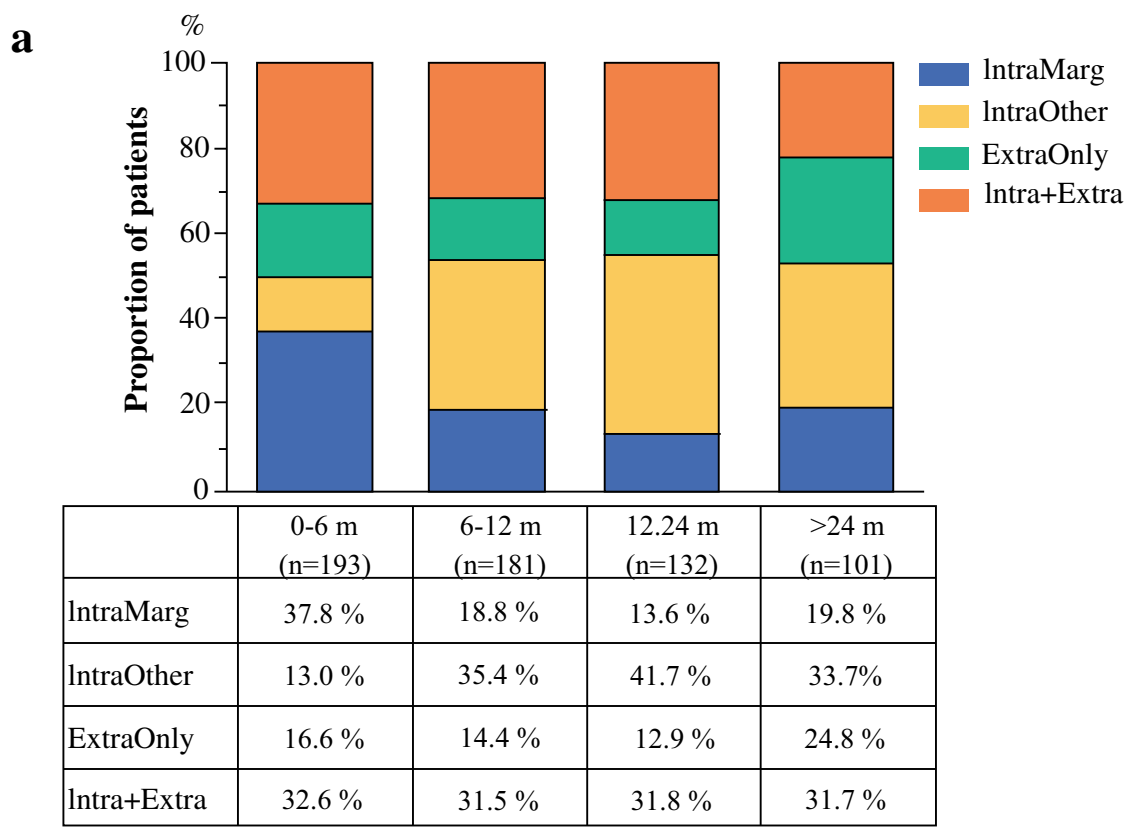

b

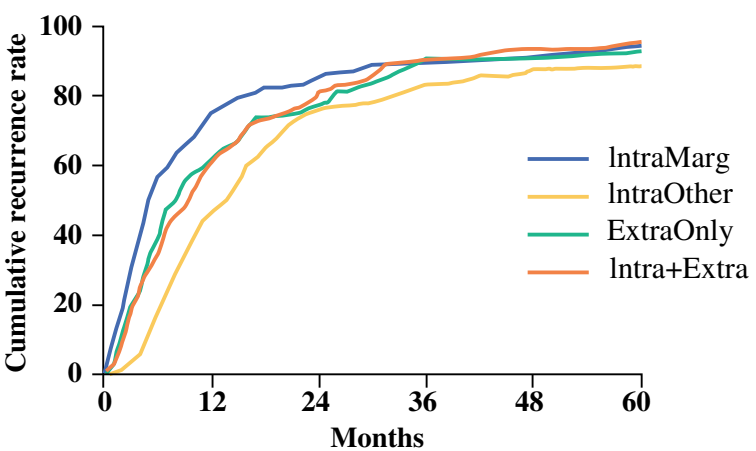

\begin{tabular}{|l|c|c|c|c|c|}
\hline Recur. patterns & $0-6 \mathrm{~m}$ & $6-12 \mathrm{~m}$ & $12-24 \mathrm{~m}$ & $24-48 \mathrm{~m}$ & $>48 \mathrm{~m}$ \\
\hline IntraMarg & $50.3 \%$ & $23.4 \%$ & $12.4 \%$ & $8.3 \%$ & $5.5 \%$ \\
\hline IntraOther & $14.0 \%$ & $36.0 \%$ & $30.9 \%$ & $11.2 \%$ & $7.9 \%$ \\
\hline ExtraOnly & $35.6 \%$ & $28.9 \%$ & $18.9 \%$ & $14.4 \%$ & $2.2 \%$ \\
\hline Intra+Extra & $32.5 \%$ & $29.4 \%$ & $21.6 \%$ & $12.9 \%$ & $3.6 \%$ \\
\hline All recurrence & $31.8 \%$ & $29.8 \%$ & $21.7 \%$ & $11.5 \%$ & $5.1 \%$ \\
\hline
\end{tabular}

FIG. 1 a Distribution of recurrence patterns at different time points. b Recurrence stratified by recurrence patterns at different time points. c Disease-free survival among patients who experienced different recurrence patterns. IntraM intrahepatic margin recurrence,

recurrence at another location in the liver was identified in 15 patients, 5 of whom also had concomitant extrahepatic recurrence.

Patients who experienced an intrahepatic margin recurrence (median DFS 6.0 months), as well as patients who recurred with extrahepatic-only recurrence (median DFS 8.9 months) had the shortest DFS (Fig. 1c). In contrast,
C

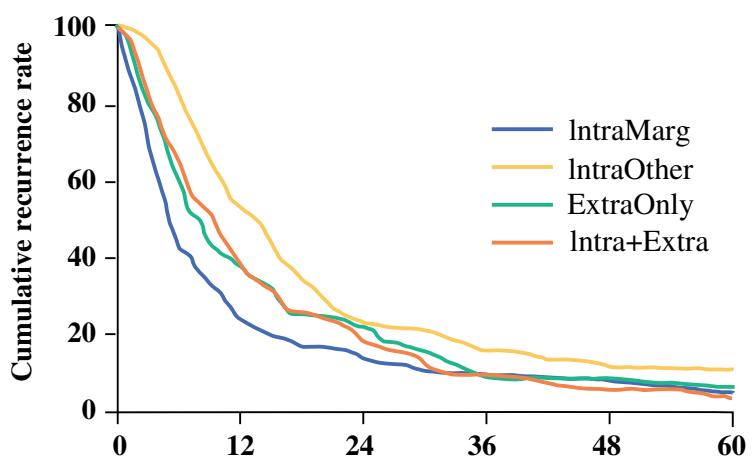

Months

\begin{tabular}{|c|c|c|c|c|c|}
\hline Recur. patterns & $\begin{array}{c}\text { Median DFS } \\
(95 \% \text { CI })\end{array}$ & lntraMarg & lntraOther & ExtraOnly & Intra+Extra \\
\hline IntraMarg & $\begin{array}{c}6.0 \\
(5.0-7.0)\end{array}$ & & $<\mathbf{0 . 0 0 1}$ & 0.144 & 0.017 \\
\hline IntraOther & $\begin{array}{c}14.4 \\
(11.4-16.6)\end{array}$ & $<\mathbf{0 . 0 0 1}$ & & $\mathbf{0 . 0 0 5}$ & $\mathbf{0 . 0 0 8}$ \\
\hline ExtraOnly & $\begin{array}{c}8.9 \\
(6.1-9.9)\end{array}$ & 0.144 & $\mathbf{0 . 0 0 5}$ & & 0.591 \\
\hline Intra+Extra & $\begin{array}{c}10.3 \\
(8.1 .11 .9)\end{array}$ & $\mathbf{0 . 0 1 7}$ & $\mathbf{0 . 0 0 8}$ & 0.591 & \\
\hline
\end{tabular}

IntraOther intrahepatic other site recurrence, ExtraOnly extrahepatic-only recurrence, Intra + Extra intrahepatic plus extrahepatic recurrence, Recur recurrence, DFS disease-free survival, $C I$ confidence interval

patients with intrahepatic recurrence that occurred at a site other than the original resection margin had the longest median DFS (14.4 months; $p<0.001)$. Of note, within 6 months following curative-intent resection, only $14 \%$ of intrahepatic other site recurrences had occurred compared with $50.3 \%$ of all intrahepatic margin recurrences having developed during this time period. In contrast, $35.6 \%$ of 
extrahepatic-only recurrence and $32.5 \%$ of intrahepatic plus extrahepatic recurrences occurred within the first 6 months following curative-intent resection (Fig. 1b). Among patients who developed tumor recurrence after surgery, patients with intrahepatic recurrence in segments other than the original resection margin had a longer median OS (51.5 months) compared with patients who experienced an intrahepatic margin recurrence (median OS 18.8 months; $p<0.001$; electronic supplementary Fig. 1).

\section{Risk Factors Associated with Different Recurrence Patterns}

In assessing the entire cohort on multivariate analysis, specific tumor characteristics such as lymph node metastasis (HR 1.22, 95\% CI 1.13-1.31; $p<0.001$ ), tumor size $>5 \mathrm{~cm}$ (HR 1.86, 95\% CI 1.39-2.50; $p<0.001)$, and multiple tumors (HR 1.66, 95\% CI 1.26-2.19; $p<0.001$ ) were associated with an increased risk of tumor recurrence after curative resection of ICC (electronic supplementary Table 1). In addition, several factors were associated with specific patterns of recurrence. For example, surgical margin $<10 \mathrm{~mm}$ (HR 2.07, 95\% CI 1.06-4.04; $p=0.034)$ and major hepatectomy (HR 2.05, 95\% CI 1.00-4.17; $p=0.049)$ were associated with an increased risk of intrahepatic margin recurrence (Table 2). Patient and liver factors such as female sex (HR 2.12, 95\% CI 1.40-3.22; $p<0.001$ ) and liver cirrhosis (HR 2.36, 95\% CI 1.31-4.25; $p=0.004)$ were both associated with an increased risk of de novo intrahepatic recurrence away from the surgical margin (Table 3), while tumor size $>5 \mathrm{~cm}$ (HR 1.82, 95\% CI $1.05-3.15 ; p=0.032$ ) was associated with risk of extrahepatic-only recurrence (electronic supplementary Table 2).

\section{Survival After Recurrence}

Median survival after recurrence was 11.3 months (95\% CI 9.6-12.4) among the 607 patients who recurred. Among these patients, 485 (79.9\%) had detailed information regarding treatment of the recurrent disease. Perhaps not surprisingly, median survival after recurrence was better among the 88 patients who underwent repeat curative-intent surgery versus the 397 patients receiving other treatments (median survival after recurrence 48.6 vs. 9.7 months; $p<0.001$; Fig. 2). Of note, 289 patients who received chemotherapy and/or radiotherapy for recurrent disease had a comparable survival after recurrence compared with the 44 patients who underwent non-curative surgery. The 49 patients who received intra-arterial therapy and the 15 patients treated with only best supportive care had the worst survival after recurrence (Fig. 2).

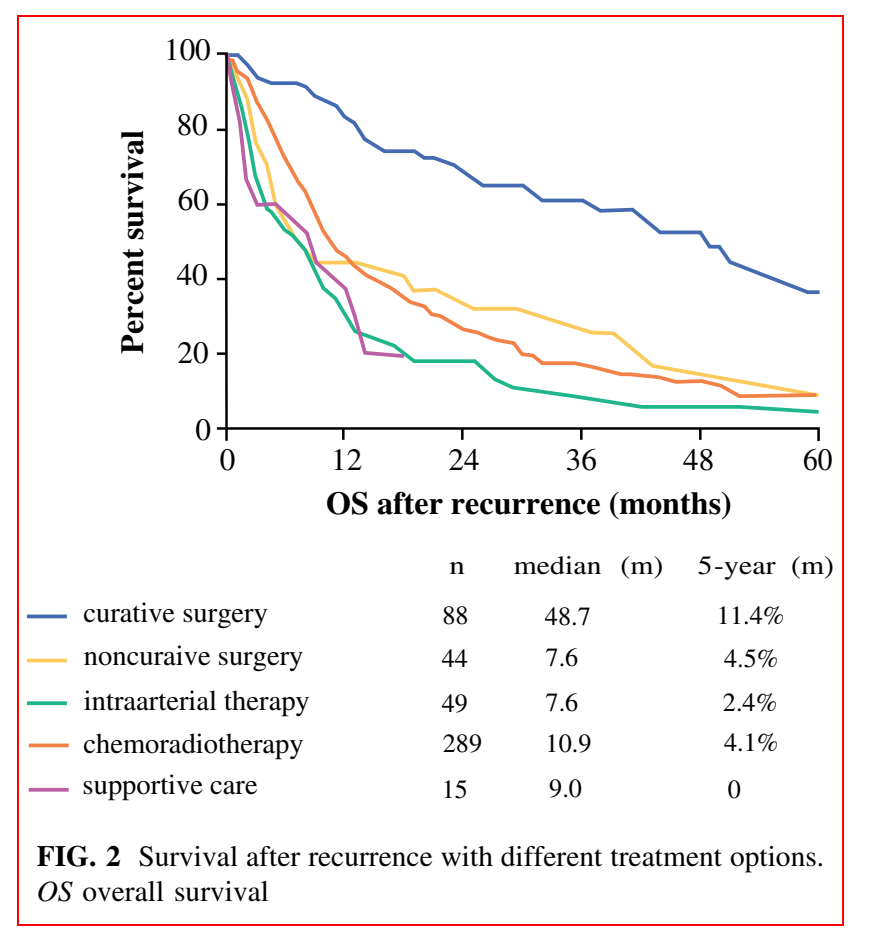

\section{DISCUSSION}

A high incidence of recurrence is one of the major factors contributing to poor outcome among patients with ICC after curative-intent surgical resection. Based on a large, international, multi-institutional database, data from the current study defined the timing course, survival impact, and risk factors associated with different patterns of recurrence following resection of ICC. Specifically, half of all recurrences at the intrahepatic surgical margin site occurred as early as 6 months after surgery. In contrast, approximately only onethird of extrahepatic-only recurrences and concomitant intrahepatic and extrahepatic recurrences occurred within 6 months after surgery. Of note, $80 \%$ of intrahepatic recurrence sites distant from the surgical margin occurred 2 years after surgery. In addition, specific recurrence patterns had a different DFS. For example, patients who had a non-surgical margin intrahepatic recurrence had a better DFS than patients with other patterns of recurrence. Furthermore, the risk factors associated with different recurrence patterns varied, suggesting possible unique biological characteristics of ICC disease based on the site of recurrence. Perhaps not surprisingly, attempts at curative-intent re-resection of the recurrence were associated with better outcomes versus patients treated with other modalities. Collectively, the data suggested that different recurrence patterns after surgical resection for ICC may reflect unique tumor biological behavior, which may impact choices about surveillance and adjuvant therapy for patients with ICC. 
TABLE 2 Risk factors of intrahepatic margin recurrence

\begin{tabular}{|c|c|c|c|c|c|}
\hline \multirow[t]{2}{*}{ Variable } & \multirow[t]{2}{*}{$N=145(\%)$} & \multicolumn{2}{|c|}{ Univariate analysis } & \multicolumn{2}{|c|}{ Multivariate analysis } \\
\hline & & $p$ value & HR $(95 \% \mathrm{CI})$ & $p$ value & $\mathrm{HR}(95 \% \mathrm{CI})$ \\
\hline Age $>65$ years & $37(25.5)$ & 0.102 & $0.72(0.48-1.07)$ & & \\
\hline Male sex & $75(51.7)$ & 0.025 & $0.67(0.47-0.95)$ & 0.771 & $0.91(0.49-1.69)$ \\
\hline Liver cirrhosis & $24(16.6)$ & 0.080 & $1.50(0.95-2.36)$ & & \\
\hline Tumor size $>5 \mathrm{~cm}$ & $102(70.3)$ & 0.451 & $1.16(0.79-1.70)$ & & \\
\hline Nodal metastasis & $35(52.2)$ & 0.047 & $1.59(1.01-2.53)$ & 0.097 & $1.14(0.98-1.32)$ \\
\hline Poorly to undifferentiated & $23(15.9)$ & 0.844 & $0.95(0.59-1.54)$ & & \\
\hline Macrovascular invasion & $14(9.7)$ & 0.737 & $0.90(0.50-1.64)$ & & \\
\hline Microvascular invasion & $39(26.9)$ & 0.326 & $1.22(0.82-1.81)$ & & \\
\hline Multiple tumors & $36(24.8)$ & 0.228 & $1.28(0.86-1.89)$ & & \\
\hline Margin $<10 \mathrm{~mm}$ & $84(57.9)$ & 0.003 & $1.82(1.22-2.72)$ & 0.034 & $2.07(1.06-4.04)$ \\
\hline Adjuvant chemo-/radiotherapy & $47(32.4)$ & 0.009 & $0.61(0.42-0.88)$ & 0.140 & $0.62(0.33-1.17)$ \\
\hline Major hepatectomy & $76(52.4)$ & 0.003 & $1.65(1.18-2.29)$ & 0.049 & $2.05(1.00-4.17)$ \\
\hline Major vascular resection & $10(6.9)$ & 0.857 & $1.06(0.56-2.02)$ & & \\
\hline Bile duct resection & $21(14.5)$ & 0.016 & $1.83(1.12-2.98)$ & 0.978 & $1.01(0.44-2.33)$ \\
\hline Periductal infiltrating \pm mass-forming & $29(20.0)$ & $<0.001$ & $1.58(1.27-1.96)$ & 0.069 & $2.22(0.94-5.24)$ \\
\hline
\end{tabular}

$H R$ hazard ratio, $C I$ confidence interval

TABLE 3 Risk factors of intrahepatic recurrence at other sites rather than margin

\begin{tabular}{|c|c|c|c|c|c|}
\hline \multirow[t]{2}{*}{ Variable } & \multirow[t]{2}{*}{$N=178(\%)$} & \multicolumn{2}{|c|}{ Univariate analysis } & \multicolumn{2}{|c|}{ Multivariate analysis } \\
\hline & & $p$ value & HR $(95 \% \mathrm{CI})$ & $p$ value & HR $(95 \% \mathrm{CI})$ \\
\hline Age $>65$ years & $67(37.6)$ & 0.876 & $0.97(0.69-1.37)$ & & \\
\hline Male sex & $99(55.6)$ & 0.022 & $1.47(1.05-2.08)$ & $<0.001$ & $2.12(1.40-3.22)$ \\
\hline Liver cirrhosis & $19(10.7)$ & 0.013 & $2.02(1.16-3.50)$ & 0.004 & $2.36(1.31-4.25)$ \\
\hline Tumor size $>5 \mathrm{~cm}$ & $116(65.2)$ & 0.781 & $1.05(0.75-1.48)$ & & \\
\hline Nodal metastasis & $25(29.1)$ & 0.526 & $1.17(0.72-1.91)$ & & \\
\hline Poorly to undifferentiated & $28(15.7)$ & 0.815 & $0.95(0.60-1.49)$ & & \\
\hline Macrovascular invasion & $13(7.3)$ & 0.361 & $1.35(0.71-2.58)$ & & \\
\hline Microvascular invasion & $40(22.5)$ & 0.516 & $1.14(0.77-1.67)$ & & \\
\hline Multiple tumors & $39(21.9)$ & 0.136 & $1.35(0.91-1.99)$ & & \\
\hline Margin $<10 \mathrm{~mm}$ & $122(68.5)$ & 0.039 & $1.62(1.03-2.56)$ & 0.103 & $1.62(1.03-2.56)$ \\
\hline Adjuvant chemo-/radio therapy & $65(36.5)$ & 0.205 & $0.80(0.57-1.13)$ & & \\
\hline Major hepatectomy & $100(56.2)$ & 0.387 & $0.88(0.65-1.18)$ & & \\
\hline Major vascular resection & $11(6.2)$ & 0.982 & $1.01(0.55-1.86)$ & & \\
\hline Bile duct resection & $26(14.6)$ & 0.449 & $0.85(0.56-1.29)$ & & \\
\hline Periductal infiltrating \pm mass-forming & $38(21.3)$ & 0.741 & $0.94(0.65-1.36)$ & & \\
\hline
\end{tabular}

Isolated intrahepatic recurrence has been reported to be among the most common sites of recurrence for ICC after curative-intent surgery. ${ }^{16}$ In fact, in the current study, $53.2 \%$ of all recurrences occurred only within the liver. Unlike previous studies, we were able to identify distinct characteristics of intrahepatic margin recurrence versus intrahepatic other site recurrence. Of note, margin recurrence occurred earlier in the postoperative course and was associated with inadequate/smaller resection margin width. Inadequate margin has previously been reported as a risk factor for tumor recurrence after resection of ICC. 19,20 Several studies reported that margin width affected long- 
term outcomes, with an incremental worsening of DFS and OS as margin width decreased. ${ }^{21,22}$ Although the definition of $\mathrm{R} 0$ resection for ICC remains controversial, ${ }^{4,19,20}$ the current study noted that a margin width of $<10 \mathrm{~mm}$ was associated with intrahepatic margin recurrence. In fact, inadequate margin could not only be a marker of technical quality but also a signature of worse disease biology. ${ }^{19-22}$

In contrast to surgical margin recurrence, recurrences in the remnant liver at sites other than the margin increased gradually, with $80 \%$ having occurred within 2 years after surgery. Intrahepatic non-margin recurrence was not associated with past surgical technique, rather de novo intrahepatic recurrence was strongly associated with patient- and liver-specific factors. In particular, consistent with previous studies, we noted that non-margin liver recurrence was associated with the presence of liver cirrhosis. 4,11, 20, 23 Recurrence as a consequence of underlying liver cirrhosis most likely represented de novo recurrence, which was different from the recurrence of the initial tumors at the surgical margin. ${ }^{16}$ Of note, among the 22 patients who developed recurrence more than 5 years after surgery, most $(68.2 \%)$ had intrahepatic recurrence at sites other than the surgical margin. Future studies should aim to define possible genetic and clonal variations in margin versus non-margin intrahepatic recurrent ICC tumors.

Another important finding of the current study was the different time courses and DFS among patients with different first site recurrence patterns. Several studies have noted that most recurrences of ICC occurred during the first 2 years after the initial surgery. ${ }^{24-26}$ As such, recurrence of ICC has been divided into early and late stages, using 24 months after surgery as the time cut-off. ${ }^{3,11}$ In the current study, when patients were stratified according to different recurrence patterns, intrahepatic margin recurrence was noted to occur earlier than other recurrence types. In addition, intrahepatic margin recurrence had the worst DFS, followed by extrahepatic-only recurrence and both intrahepatic and extrahepatic recurrence. Of note, patients with non-margin intrahepatic recurrence had the longest DFS. Close margins $(<10 \mathrm{~mm})$ were associated with early margin recurrence with short DFS. In contrast, extrahepatic-only recurrence was related to initial tumor characteristics such as tumor size $(5 \mathrm{~cm})$, which has been recognized as a prognostic risk factor in the AJCC 8th edition staging system of ICC. ${ }^{27,28}$

Management of recurrent ICC remains a clinical challenge. Repeat curative resection for recurrent ICC has been associated with improved outcomes compared with adjuvant chemotherapy or best supportive care.,8,29 In the current study, median survival after recurrence was indeed better among patients who underwent repeat curative-intent surgery versus other treatments. As such, repeat surgery can be considered in the small subset of patients with limited recurrent disease who have good tumor biology. Unfortunately, most patients with recurrent disease will not be candidates for repeat resection. These patients with unresectable recurrent ICC may benefit from systematic adjuvant chemotherapy. ${ }^{30-32}$

The current study had several limitations. The retrospective study design may have led to inherent selection bias, given that only patients who underwent a resection were included in the study. While the multi-institutional collaboration undoubtedly increased the sample size, there were likely some discrepancies in patient selection, followup, and adjuvant therapies among the centers. While surveillance practices of different centers may have varied somewhat, the majority of patients had regular follow-up as noted. However, it is likely that a subset of patients who had suspicious imaging or laboratory findings and underwent more frequent imaging would be more likely to be diagnosed with recurrence earlier.

\section{CONCLUSIONS}

The current study provided detailed information on timing, patterns, and risk factors associated with different recurrence patterns after surgery for ICC. Intrahepatic margin recurrence was likely to be a recurrence due to residue tumor at the surgical margin as this pattern of recurrence was associated with an initial narrow $(<10 \mathrm{~mm})$ surgical margin. Surgical margin recurrence occurred early and was associated with the worse DFS. In contrast, non-surgical margin intrahepatic recurrence was more likely to occur later in the natural history of the patient's postoperative course. The fact that non-margin recurrence was associated with underlying liver disease and took a longer time to develop suggested that this manifestation of disease was likely de novo disease rather than 'true' recurrence. The different recurrence patterns and timing of recurrence suggest biological heterogeneity of ICC tumor recurrence. In turn, understanding the timing and risk factors associated with the different types of recurrence can hopefully inform discussions around adjuvant therapy, surveillance, and treatment of recurrent disease.

DISCLOSURES Liang-Shuo Hu, Xu-Feng Zhang, Matthew Weiss, Irinel Popescu, Hugo P. Marques, Luca Aldrighetti, Shishir K. Maithel, Carlo Pulitano, Todd W. Bauer, Feng Shen, George A. Poultsides, Oliver Soubrane, Guillaume Martel, B. Groot Koerkamp, Endo Itaru, and Timothy M. Pawlik have no financial or commercial interests to disclose. 


\section{REFERENCES}

1. Amini N, Ejaz A, Spolverato G, Kim Y, Herman JM, Pawlik TM. Temporal trends in liver-directed therapy of patients with intrahepatic cholangiocarcinoma in the United States: a populationbased analysis. J Surg Oncol. 2014;110:163-70.

2. Hyder O, Hatzaras I, Sotiropoulos GC, et al. Recurrence after operative management of intrahepatic cholangiocarcinoma. Surgery. 2013;153:811-8.

3. Doussot A, Gonen M, Wiggers JK, et al. Recurrence patterns and disease-free survival after resection of intrahepatic cholangiocarcinoma: preoperative and postoperative prognostic models. $J$ Am Coll Surg. 2016;223:493-505 e2.

4. Spolverato G, Kim Y, Alexandrescu S, et al. Management and outcomes of patients with recurrent intrahepatic cholangiocarcinoma following previous curative-intent surgical resection. Ann Surg Oncol. 2016;23:235-43.

5. Sulpice L, Rayar M, Boucher E, Pracht M, Meunier B, Boudjema $\mathrm{K}$. Treatment of recurrent intrahepatic cholangiocarcinoma. $\mathrm{Br} \mathrm{J}$ Surg. 2012;99:1711-7.

6. Wang Y, Li J, Xia Y, et al. Prognostic nomogram for intrahepatic cholangiocarcinoma after partial hepatectomy. J Clin Oncol. 2013;31:1188-95.

7. Spolverato G, Vitale A, Cucchetti A, et al. Can hepatic resection provide a long-term cure for patients with intrahepatic cholangiocarcinoma? Cancer. 2015;121:3998-4006.

8. Ercolani G, Vetrone G, Grazi GL, et al. Intrahepatic cholangiocarcinoma: primary liver resection and aggressive multimodal treatment of recurrence significantly prolong survival. Ann Surg. 2010;252:107-14.

9. Tabrizian P, Jibara G, Shrager B, Schwartz M, Roayaie S. Recurrence of hepatocellular cancer after resection: patterns, treatments, and prognosis. Ann Surg. 2015;261:947-55.

10. Hirokawa F, Hayashi M, Asakuma M, Shimizu T, Inoue Y, Uchiyama K. Risk factors and patterns of early recurrence after curative hepatectomy for hepatocellular carcinoma. Surg Oncol. 2016;25:24-9.

11. Zhang XF, Beal EW, Bagante F, et al. Early versus late recurrence of intrahepatic cholangiocarcinoma after resection with curative intent. Br J Surg. 2018;105:848-56.

12. Poon RT, Fan ST, Ng IO, Lo CM, Liu CL, Wong J. Different risk factors and prognosis for early and late intrahepatic recurrence after resection of hepatocellular carcinoma. Cancer. 2000;89: 500-7.

13. Imamura H, Matsuyama $\mathrm{Y}$, Tanaka $\mathrm{E}$, et al. Risk factors contributing to early and late phase intrahepatic recurrence of hepatocellular carcinoma after hepatectomy. $J$ Hepatol. 2003;38:200-7.

14. Portolani N, Coniglio A, Ghidoni S, et al. Early and late recurrence after liver resection for hepatocellular carcinoma: prognostic and therapeutic implications. Ann Surg. 2006;243: 229-35.

15. Cucchetti A, Piscaglia F, Caturelli E, et al. Comparison of recurrence of hepatocellular carcinoma after resection in patients with cirrhosis to its occurrence in a surveilled cirrhotic population. Ann Surg Oncol. 2009;16:413-22.

16. Zhang XF, Beal EW, Chakedis J, et al. Early Recurrence of neuroendocrine liver metastasis after curative hepatectomy: risk factors, prognosis, and treatment. J Gastrointest Surg. 2017; 21(11): 1821-30.

17. Amin MB. American Joint Committee on Cancer. AJCC cancer staging manual. Chicago: Springer; 2017.
18. Okusaka T, Okada S, Ueno H, et al. Satellite lesions in patients with small hepatocellular carcinoma with reference to clinicopathologic features. Cancer. 2002;95:1931-7.

19. Li MX, Bi XY, Li ZY, et al. Impaction of surgical margin status on the survival outcome after surgical resection of intrahepatic cholangiocarcinoma: a systematic review and meta-analysis. $J$ Surg Res. 2016;203:163-73.

20. Mavros MN, Economopoulos KP, Alexiou VG, Pawlik TM. Treatment and prognosis for patients with intrahepatic cholangiocarcinoma: systematic review and meta-analysis. JAMA Surg. 2014;149:565-74.

21. Zhang XF, Bagante F, Chakedis J, et al. Perioperative and longterm outcome for intrahepatic cholangiocarcinoma: impact of major versus minor hepatectomy. J Gastrointest Surg. 2017;21: 1841-50.

22. Spolverato G, Yakoob MY, Kim Y, et al. The impact of surgical margin status on long-term outcome after resection for intrahepatic cholangiocarcinoma. Ann Surg Oncol. 2015;22:4020-8.

23. Hyder O, Marques H, Pulitano C, et al. A nomogram to predict long-term survival after resection for intrahepatic cholangiocarcinoma: an Eastern and Western experience. JAMA Surg. 2014;149:432-8.

24. Tarchi P, Tabrizian P, Prigoff J, Schwartz M. Outcomes of resection for solitary $\leq 5 \mathrm{~cm}$ intrahepatic cholangiocarcinoma. Surgery. 2018;163:698-702.

25. Zhang XF, Chakedis J, Bagante F, et al. Implications of intrahepatic cholangiocarcinoma etiology on recurrence and prognosis after curative-intent resection: a multi-institutional study. World $J$ Surg. 2018;42:849-57.

26. Buettner S, Galjart B, van Vugt JLA, et al. Performance of prognostic scores and staging systems in predicting long-term survival outcomes after surgery for intrahepatic cholangiocarcinoma. J Surg Oncol. 2017;116:1085-95.

27. Kim Y, Moris DP, Zhang XF, et al. Evaluation of the 8th edition American Joint Commission on Cancer (AJCC) staging system for patients with intrahepatic cholangiocarcinoma: A surveillance, epidemiology, and end results (SEER) analysis. J Surg Oncol. 2017;116(6):643-50.

28. Spolverato G, Ejaz A, Kim Y, et al. Tumor size predicts vascular invasion and histologic grade among patients undergoing resection of intrahepatic cholangiocarcinoma. J Gastrointest Surg. 2014;18:1284-91.

29. Si A, Li J, Xing X, et al. Effectiveness of repeat hepatic resection for patients with recurrent intrahepatic cholangiocarcinoma: factors associated with long-term outcomes. Surgery. 2017;161: 897-908.

30. Chun YS, Javle M. Systemic and adjuvant therapies for intrahepatic cholangiocarcinoma. Cancer Control. 2017;24:10732748 17729241.

31. Valle J, Wasan H, Palmer DH, et al. Cisplatin plus gemcitabine versus gemcitabine for biliary tract cancer. $N$ Engl J Med. 2010;362:1273-81.

32. Mizusawa J, Morizane C, Okusaka T, et al. Randomized phase III study of gemcitabine plus S-1 versus gemcitabine plus cisplatin in advanced biliary tract cancer: Japan Clinical Oncology Group Study (JCOG1113, FUGA-BT). Jpn J Clin Oncol. 2016;46: $385-8$.

Publisher's Note Springer Nature remains neutral with regard to jurisdictional claims in published maps and institutional affiliations. 